Pacific Journal of Mathematic 


\title{
A SOBOLEV SPACE AND A DARBOUX PROBLEM
}

\author{
M. B. SURyanarayana
}

This paper deals with a class of functions which are defined in an $n$-dimensional rectangle and which possess there, only the generalized partial derivatives of mixed type. It is shown that (i) this class contains as a proper subset the usual Sobolev class of order $n$, the dimension of the domain and (ii) this class can be imbedded in the space of continuous functions. In addition to the compactness of the imbedding operator, the closedness of certain nonlinear partial integro differential operators is also studied. Finally, a system of partial integro differential equations with Darboux type boundary data in a rectangle, is shown to have solutions in this class. The results of this paper are used in certain existence theorems of optimal control theory.

1. Introduction. In recent studies on existence theorems for optimization problems involving Darboux type side conditions, it was found useful and necessary to introduce a special class of functions, (see for example [11] and [7]). This special class which we shall denote as $W_{p}^{*}(G), G \subset E^{n}$, consists of all functions $z(t), t \in G$, with $z \in L_{p}(G)$, and such that $z$ has all (and only) the mixed partial generalized derivatives $D_{\alpha} z$ of orders upto and including $n$ (the number of independent variables) with $D_{\alpha} z \in L_{p}(G)$; thus, derivatives of $z$ taken more than once with respect to any of the variables $t_{1}, \cdots, t_{n}$, may not even exist. In the case of $n=2$, for example, with independent variables $x$ and $y$, this would mean that for $z \in W_{p}^{*}(G)$, the generalized partials $z_{x}, z_{y}$ and $z_{x y}$ exist and belong to $L_{p}(G)$ while the pure partials $z_{x x}$ and $z_{y y}$ need not even exist. This class is thus analogous to the classical $C^{*}(G)$ where only the derivatives $z_{x}, z_{y}$ and $z_{x y}$ exist and are continuous.

Clearly, $W_{p}^{*}(G)$ contains $W_{p}^{n}(G)$, the usual Sobolev class of functions for which all the generalized partial derivatives of order upto and including $n$ exist and belong to $L_{p}(G)$. We shall show in no. 2 below that there exist functions in $W_{p}^{*}(G)$ which are not in $W_{p}^{n}(G)$. One of the purposes of this paper is to analyze this special class of functions $W_{p}^{*}(G)$ for $G=[a, a+h] \subset E^{n}, n \geqq 1$ and in particular to show that it can be imbedded in $C(G)$, the space of continuous functions, for all $p, 1 \leqq p \leqq \infty$. In particular, it follows that $W_{p}^{n}(G) \subset C(G)$ even for $p=1$. We shall also study criteria for the compactness of the imbedding operator. In the same context, the closedness of some nonlinear integro differential operators is investigated, a result used in the closure theorems relevant to the 
existence theory of optimal control problems (see [11]).

The last section of this paper is devoted to establish an existence and uniqueness theorem in the special class mentioned above, for solutions of a system of partial integro differential equations with Darboux type boundary data in a rectangle in $E^{n}$. This theorem is derived as a consequence of the existence theorems for multidimensional integral equations of Volterra type given by the author in [9]. Since the Darboux problem considered here involves a control function also, the existence of solutions for each measurable control under the specified hypothesis), therefore proves the controllability of the system. These results are used in [11].

Examples are provided through out the paper to illustrate the statements.

2. Notations. Let $E^{n}, n \geqq 1$ denote the $n$-dimensional Euclidean space. Let

$$
\begin{aligned}
G & =[a, a+h] \\
& =\left\{t \in E^{n} \mid t=\left(t_{1}, \cdots, t_{n}\right), a_{i} \leqq t_{i} \leqq a_{i}+h_{i}, i=1, \cdots, n\right\} .
\end{aligned}
$$

Let $C(G)$ denote the space of continuous functions on $G$, as usual with the supremum norm and let $L_{p}(G)$ be the space of all measurable functions whose $p$-th powers are integrable over $G$, for $1 \leqq p<\infty$. Let $L_{\infty}(G)$ be the space of all essentially bounded functions measurable on $G$.

Let $T_{i}$ denote the operator defined on $X^{m}, m \geqq 1$ (with $X=C(G)$, $L_{p}(G)$ or $\left.L_{\infty}(G)\right)$ as follows:

$$
T_{i} z(t)=\int_{a_{i}}^{t_{i}} z\left(t_{i}^{\prime}, s\right) d s, \quad z \in X^{m} .
$$

Here $t_{i}^{\prime}=\left(t_{1}, \cdots, t_{i-1}, t_{i+1}, \cdots, t_{n}\right)$ so that $t=\left(t_{i}, t_{i}^{\prime}\right)$. We define $T_{i} T_{j}$ as composition so that $T_{i}^{r} z=T_{i}\left(T_{i}^{r-1} z\right), r \geqq 1$ and $T_{i}^{0} z=z$ for $z \in X^{m}$. Let $D_{i} z$ denote as usual the generalized derivative of $z$ with respect to $t_{i}, i=1, \cdots, n$.

A mutiindex $\alpha=\left(\alpha_{1}, \cdots, \alpha_{n}\right)$ is an $n$-vector with arbitrary nonnegative integers $\alpha_{i}, i=1, \cdots, n$. As usual $|\alpha|=\alpha_{1}+\cdots+\alpha_{n}$ and $\alpha !=\alpha_{1} ! \cdots \alpha_{n} !$. Let $J$ denote the set of all multiindices $\alpha$ with $|\alpha|<n$ and $\alpha_{i}=0$ or 1 for $i=1, \cdots, n$. Let $\bar{J}=J \cup\{(1, \cdots, 1)\}$. In the sequel, unless specified otherwise, multiindices (denoted by greek letters) are from $\bar{J}$. Also $\alpha=1$ shall mean $\alpha_{i}=1$ for all $i$ while $|\alpha|=1$ means $\alpha_{i}=1$ for one and only one $i$. For $\alpha, \beta \in \bar{J}$, the inner product $\Sigma \alpha_{i} \beta_{i}$ is denoted by $\alpha \cdot \beta$ and $\alpha^{\prime}$ denotes $1-\alpha$; that is $\alpha_{i}^{\prime}=1-\alpha_{i}, i=1, \cdots, n$. By $\alpha \leqq \beta$ we shall mean $\alpha_{i} \leqq \beta_{i}$ for each $i$ while $\alpha<\beta$ means $\alpha \leqq \beta$ and $\alpha \neq \beta$. Thus, for example, 
$(1,0,1)<(1,1,1)$

Given $\beta \in J$, there are just as many $\pi \in \bar{J}, \pi \cdot \beta=0$ with $|\pi|$ even, as there are with $|\pi|$ odd (the total number being $\left.2^{n-|\beta|}\right)$. So, $\Sigma(-1)^{|\pi|}=-1$ where the summation is taken over all $\pi \in \bar{J}-\{0\}$ with $\pi \cdot \beta=0$. The following lemma is now an immediate consequence of this.

LEMMA 1. Given any real valued function $f(\beta)$ on $\bar{J}$ and $\alpha \in \bar{J}$ we have

$$
\sum_{\substack{0 \leq \beta<\alpha \\ \beta \in J}} f(\beta)=\sum_{i=1}^{|\alpha|} \sum_{\substack{0<\pi \leq \alpha \\|\pi|=i \\ \pi \in J}} \sum_{\substack{\pi \cdot \beta=0 \\ 0 \leq \beta<\alpha \\ \beta \in J}}(-1)^{i-1} f(\beta) .
$$

REMARK. The above formula is that of the cardinality of union of a collection of sets.

Illustration. If $\alpha=(1,0,1,0)$ then $0 \leqq \beta<\alpha$ is satisfied by $\beta_{1}=(0,0,0,0), \beta_{2}=(1,0,0,0)$ and $\beta_{3}=(0,0,1,0)$. Also, $0<\pi \leqq \alpha$ is satisfied by $\pi_{1}=(1,0,0,0), \pi_{2}=(0,0,1,0)$ and $\pi_{3}(1,0,1,0)$. In this case, $\pi_{1} \cdot \beta_{1}=\pi_{1} \cdot \beta_{3}=0 ; \pi_{2} \cdot \beta_{1}=\pi_{2} \cdot \beta_{2}=0$ and $\pi_{3} \cdot \beta_{1}=0$ so that the right hand side of the equation in the lemma is

$$
f\left(\beta_{1}\right)+f\left(\beta_{3}\right)+f\left(\beta_{1}\right)+f\left(\beta_{2}\right)-f\left(\beta_{1}\right)=\sum_{i=1}^{3} f\left(\beta_{i}\right)=\sum_{0 \leq \beta<\alpha} f(\beta) .
$$

In the sequel, multiindices from $\bar{J}$ (that is with $\alpha_{i}=0$ or 1 for all $i$ ) appear as subscripts in three different ways: (1) As subscripts of the set $G$ or a point in $G$, they mean projection into the corresponding coordinates. Thus $t_{\alpha}$ means the $|\alpha|$-vector obtained omitting those $t_{i}$ in $\left(t_{1}, \cdots, t_{n}\right)$ for which $\alpha_{i}=0$. Also

$$
G_{\alpha}=\left\{t_{\alpha} \in E^{|\alpha|}: a_{\alpha} \leqq t_{\alpha} \leqq a_{\alpha}+h_{\alpha}\right\} \text {. }
$$

If $\alpha=0$, that is $\alpha_{i}=0$ for all $i$ then $G_{\alpha}$ is the empty set. (2) As subscripts of an operator ( $T$ or $D$ ) we mean the composition $T_{1}^{\alpha_{1}} \cdots T_{n}^{\alpha_{n}}$ of those $T_{i}$ for which $\alpha_{i} \neq 0$. If $\alpha=0$ then $T_{\alpha}=T_{0}$ and $D_{\alpha}=D_{0}$ are understood as identity operators; Thus $T_{0} z=D_{0} z=z$. In either of the two cases (1) and (2), a prime' on the top shall indicate the complementary index. Thus $T_{\alpha}^{\prime}=T_{\alpha^{\prime}}=T_{1-\alpha}$. For $n=3$, and $\alpha=(1,0,1)$ for example, $T_{\alpha}=T_{1} T_{3}$ while $T_{\alpha}^{\prime}=T_{2}$. (3) As subscripts of functions, no further meaning is attached. Thus, $R_{\alpha}(t)$ is just the $\alpha$ th function on $G$.

For $1 \leqq k \leqq n$ if $k$ is the subscript of an operator ( $T$ or $D$ ) then it is same as having a subscript $\alpha$ with $\alpha_{i}=1$ for $1 \leqq i \leqq k$ and $\alpha_{i}=0$ for $i>k$. Thus, $D^{n} z=D_{\alpha} z$ with $\alpha=(1, \cdots, 1)$.

For $1 \leqq p \leqq \infty$, let $W_{p}^{n}(G)$ denote as usual (see Sobolev [8] or 
Morrey [5]) the set of all $z \in L_{p}(G)$ with $D_{\alpha} z \in L_{p}(G)$ for all $\alpha$ with $|\alpha| \leqq n ;(\alpha$ may not be in $J)$ We define the space $W_{p}^{*}(G)$ as the set of all $z \in L_{p}(G)$ for which $D_{\alpha} z \in L_{p}(G)$ for $\alpha \in \bar{J}$. The norm on $W_{p}^{*}(G)$ is defined by $\|z\|^{*}=\sum_{\alpha \epsilon \bar{J}}\left\|D_{\alpha} z\right\|_{p}$ where $\|\cdot\|_{p}$ is the usual $L_{p}$-norm.

It may be recalled that $W_{p}^{n}(G)$ is normed by $\sum_{|\alpha| \leqq n}\left\|D_{\alpha} z\right\|_{p}$. Also, by definition, $W_{p}^{n}(G) \subset W_{p}^{*}(G)$. However, as following example shows, $W_{p}^{*}(G)$ is certainly a larger class than $W_{p}^{n}(G)$. Indeed, in the case $n=2$, and $G=[0,1] \times[0,1]$, if $z(x, y)=\int_{0}^{x} c(\alpha) d \alpha+\int_{0}^{y} c(\beta) d \beta$ where $c($.$) is the Cantor function on [0,1]$ then $z \in W_{p}^{*}(G)-W_{p}^{2}(G)$ because $z_{x x}$ does not exist (which follows from the fact that the Cantor function is not absolutely continuous.)

We define the weak convergence in $W_{p}^{*}(G)$ analogous to $W_{p}^{n}(G)$.

3. Imbedding theorem. It is well known that $W_{p}^{k}(G) \subset C(G)$ if $k p>n$. In particular, if $p>1$, then $W_{p}^{n}(G) \subset C(G)$. In this section, we show that $W_{p}^{*}(G) \subset C(G)$ for all $p, 1 \leqq p \leqq \infty$. In particular then, $W_{p}^{n}(G) \subset W_{p}^{*}(G) \subset W_{1}^{*}(G) \subset C(G)$, where inclusions are understood as imbeddings. It is to be noted that in view of this, $W_{p}^{n}(\dot{G}) \subset C(G)$ even for $p=1$. This result is analogous to Theorem 2.2.7 of Hormander [4], but of course here for bounded rectangles.

THEOREM 1. Let $G=[a, a+h] \subset E^{n}$. Then for each $z \in W_{1}^{*}(G)$, there exists $a \bar{z} \in C(G)$ such that $z=\bar{z}$ a.e. in $G$.

Proof. Since $z \in W_{1}^{*}(G), D^{n} z \in L_{1}(G)$ where $D^{n}$ denotes $D_{\alpha}$ with $\alpha=1$. Let $w \in L_{1}(G)$ be defined by

$$
w(t)=\int_{a}^{t} D^{n} z(s) d s=T^{n} D^{n} z
$$

By Fubini's theorem $w \in C(G)$. Also $w$ has generalized derivatives $D_{i} w$ given by

$$
D_{i} w(t)=D_{i} w\left(t_{i}, t_{i}^{\prime}\right)=\int_{a_{i}^{\prime}}^{t_{i}^{\prime}} D^{n} z\left(t_{i}, s\right) d s .
$$

It is seen similarly that for $\alpha \in \bar{J}, D_{\alpha} w$ exists and is given by $\int_{a_{\alpha}^{\prime}}^{t_{\alpha}^{\prime}} D^{n} z\left(t_{\alpha}, s\right) d s$. Furthermore, $D^{n} z=D^{n} w$. Now, $D^{n-1} z$ and $D^{n-1} w$ are both absolutely continuous and have the same generalized derivative with respect to $t_{n}$. Thus, (see [8, p. 21]) there is a function $c_{1, n}\left(t_{n}^{\prime}\right)$ of $t_{n}^{\prime}$ alone such that $D^{n-1}(z-w)\left(t_{n}, t_{n}^{\prime}\right)=c_{1, n}\left(t_{n}^{\prime}\right)$ for almost all $t_{n}^{\prime}$ and almost all $t_{n}$. Since $D^{n-1}(z-w)$ is in $L_{1}\left(G_{n}^{\prime}\right)$ for almost all $t_{n}$, same is true for $c_{1, n}\left(t_{n}^{\prime}\right)$. Repeating the process (see illustration below), it is seen that for $\alpha \neq 0$, 


$$
D_{\alpha}^{\prime}(z-w)(t)=\sum \int_{a_{\beta}}^{t_{\beta}} c_{|r|, \delta}\left(a_{\theta}, s_{\beta}, t_{\alpha}^{\prime}\right) d s_{\beta}
$$

where the summation in taken over all multiindices $\beta, \gamma, \delta, \theta$ with $\delta+\theta=\gamma, \gamma+\beta=\alpha ;|\delta|=1 ;|\gamma| \geqq 1 ; \beta \geqq 0 ; \theta \geqq 0$. If $\beta=0$, it is understood that there is no integration and only one (any of $|\alpha|=|\gamma|$ choices) pair $\delta, \theta$ is chosen with $\delta+\theta=\gamma=\alpha$. If $|\alpha|=1$, that is $\alpha_{i}=1$ for one and only one $i$, then clearly $\beta=0$.

If $\alpha=1$, that is $\alpha_{i}=1$ for all $i$, then $D_{\alpha}^{\prime}(z-w)=z-w$ and thus (3.3) yields

$$
z(t)=w(t)+\sum \int_{a_{\beta}}^{t_{\beta}} c_{|\gamma|, \delta}\left(a_{\theta}, s_{\beta}\right) d s_{\beta}
$$

where summation is taken as above with $\alpha=1$. It follows that $z(t)$ is equal to a continuous function a.e. in $G$.

Illustration. Let $n=3,\left(t_{1}, t_{2}, t_{3}\right)=(x, y, u)$ and $\alpha=(0,1,1)$; then $\alpha^{\prime}=(1,0,0)$. Let us consider the set of multiindices $\beta, \gamma, \delta, \theta$ to be used in formula (3.3) for this $\alpha$. Since $|\delta|=1$ and $\delta \leqq \gamma \leqq \alpha, \gamma$ can be either $\gamma_{1}=(0,0,1), \dot{\gamma}_{2}=(0,1,0)$ or $\gamma_{3}=(0,1,1)$ while $\delta$ has to be $\delta_{1}=(0,0,1)$ for $\gamma_{1}, \delta_{2}=(0,1,0)$ for $\gamma_{2}, \delta_{3}=(0,1,0)$ for $\gamma_{3}$ or $\delta_{4}=(0,0,1)$ also for $\gamma_{3}$. Then $\beta_{1}=\gamma_{2}$ for $\gamma_{1}, \beta_{2}=\gamma_{1}$ for $\gamma_{2}$ and $\beta_{3}=(0,0,0)$ for $\gamma_{3}$. In the last case, that is with $\gamma_{3}, \theta$ can take only one value: either $\theta=(0,0,1)$ with $\gamma_{3}, \delta_{3}$ or $\theta=(0,1,0)$ with $\gamma_{3}, \delta_{4}$. Thus (3.3) yields

$$
(z-w)_{x}=\int_{a_{2}}^{y} c_{1, \delta_{1}}(x, s) d s+\int_{a_{3}}^{u} c_{1, \delta_{2}}(x, t) d t+c_{2, \delta_{3}}\left(a_{3}, x\right)
$$

or a similar equation with the last term on the right hand side replaced by $c_{2, \delta_{4}}\left(a_{2}, x\right)$. Here, $w=\int_{a_{1}}^{x} \int_{a_{1}}^{y} \int_{a_{3}}^{u} z_{x y u}$. It is seen that we arrive at (3.5) by observing that since $z_{x y u}=w_{x y u}$, we have on integrating out $u, z_{x y}=w_{x y}+c_{1, \delta_{1}}(x, y)$ which becomes

$$
z_{x}=w_{x}+\int_{a_{2}}^{y} c_{1, \delta_{1}}(x, s) d s+\widetilde{c}(x, u) .
$$

Similarly, starting from $z_{x y u}=w_{x y u}$ and integrating $y$, we get $z_{x u}=w_{x u}+c_{1, \delta_{2}}(x, u)$ which becomes

$$
z_{x}=w_{x}+\int_{a_{3}}^{u} c_{1, \delta_{2}}(x, t) d t+\widetilde{\widetilde{c}}(x, y) .
$$

Equation (3.6) and (3.7) (as well as (3.3) and (3.4)) are valid for $y=a_{2}$ and $u=a_{3}$ (as boundary values). Substitution and comparison yields (3.5). 


\section{REMARKS.}

1. There exist sets $H_{i} \subset\left[a_{i}, a_{i}+h_{\imath}\right]$ with meas $\left(H_{i}\right)=0$, $i=1,2, \cdots, n$ such that formulae (3.3) and (3.4) are valid for $t \in\left[G-\bigcup_{i=1}^{n}\left(G_{i}^{\prime} \times H_{i}\right)\right]$. It is to be noted that this is stronger than saying that the equations are valid a.e. in $G$. However, the equations are valid for $\left(a_{\pi}, t_{\pi}^{\prime}\right)$ for almost all, $t_{\pi}^{\prime} \in G_{\pi}^{\prime}, 1 \leqq|\pi| \leqq n$.

2. The function $\left(D_{\alpha}^{\prime} z\right)(t)=\left(D_{\alpha}^{\prime} z\right)\left(t_{n}, t_{*}^{\prime}\right)$ is equal to a continuous function in $t_{\pi}$ for $0<\pi \leqq \alpha$.

3. Formula (3.3) can be written more explicity as follows:

$$
D_{\alpha}^{\prime}(z-w)(t)=\sum_{\substack{0 \leq \beta<\alpha \\ \beta \in J}} \int_{a_{\beta}}^{t_{\beta}} c_{\mid \alpha-\beta, \delta}\left(\alpha_{\alpha-\beta-\delta}, s_{\beta}, t_{\beta}^{\prime}\right) d s_{\beta} .
$$

If $\pi \in \bar{J}$ (and $\alpha \neq 0$ as before)

$$
D_{\alpha}^{\prime}(z-w)\left(a_{\pi}, t_{\pi}^{\prime}\right)=\sum \int_{\alpha_{\beta}}^{t_{\beta}} c_{|\alpha-\beta|, \hat{o}}\left(a_{\sigma}, s_{\beta}, t_{\rho}\right) d s_{\beta}
$$

where summation is taken over all $\beta$ with $\beta \in J, 0 \leqq \beta<\alpha$ and $\pi \cdot \beta=0 ;$ and where $\sigma=\alpha-\beta-\delta+\left(\pi \cap \alpha^{\prime}\right)$ and $\rho=\alpha^{\prime}-\left(\pi \cap \alpha^{\prime}\right)$ with $\left(\alpha^{\prime}\right)_{i}=1-\alpha_{i}$ for all $i$ and $\left(\pi \cap \alpha^{\prime}\right)_{i}=1$ if $\pi_{\imath}=\alpha_{i}^{\prime}=1$ and $\left(\pi \cap \alpha^{\prime}\right)_{i}=0$ otherwise. In particular, if $\pi \cap \alpha^{\prime}=0$ then the integrands in (3.8) and (3.9) are the same, so that using Lemma 1, no. 2 yields

$$
\begin{aligned}
D_{\alpha}^{\prime}(z-w)(t) & =\sum(-1)^{i-1} D_{\alpha}^{\prime}(z-w)\left(a_{\pi}, t_{\alpha}^{\prime}\right) \\
& =\sum(-1)^{i-1} D_{\alpha}^{\prime} z\left(a_{\pi}, t_{\pi}^{\prime}\right)
\end{aligned}
$$

because $D_{\alpha}^{\prime} w\left(\alpha_{\pi}, t_{\pi}^{\prime}\right)=0$ for $\pi \cap \alpha^{\prime}=0$. The summations in (3.10) are taken over all $i, \pi$ with $1 \leqq i \leqq|\alpha|$ and $\pi \in \bar{J},|\pi|=i, \pi \cdot \alpha^{\prime}=0$. (Let us recall that $w=\int_{a}^{t} D^{n} z(s) d s$.) In particular, if $\alpha=1$ (that is $\alpha_{i}=1$ for all $i$ ) then

$$
(z-w)(t)=\sum_{i=1}^{n} \sum_{\substack{|\pi|=-2 \\ \pi \in \bar{J}}}(-1)^{i-1} z\left(a_{\pi}, t_{\pi}^{\prime}\right)
$$

If $\pi=\alpha^{\prime}$ then $\rho=0$ and (3.9) reduces to

$$
D_{\alpha}^{\prime}(z-w)\left(a_{\alpha}^{\prime}, t_{\alpha}\right)=\sum \int_{\alpha_{\beta}}^{t_{\beta}} c_{|\alpha-\beta|, \delta}\left(\alpha_{1-\beta-\delta}, s_{\beta}\right) d s_{\beta}
$$

where 1 stands for $(1, \cdots, 1)$ and the summation is over $\beta \in J$ with $0 \leqq \beta<\alpha$ and $|\delta|=1, \delta \in J$. On the other hand from (3.9) with $\alpha=1$ we get,

$$
(z-w)\left(a_{\pi}, t_{\pi}^{\prime}\right)=\int_{a_{\beta}}^{t_{\beta}} c_{|\gamma|, \delta}\left(a_{\theta}, s_{\beta}\right) d s_{\beta}
$$

where the summation is over all $\beta \in J$ with $0 \leqq \beta<1$ and $\pi \cdot \beta=0$ and 
where $\gamma=1-\beta$ while $\theta=1-\beta-\delta$. (In all these, 1 as before stands for $(1, \cdots, 1)$.) Once again using Lemma 1 , no. 2 we get

$$
z(t)=w(t)+\sum(-1)^{i-1} D_{\alpha}^{\prime} z\left(a_{\alpha}^{\prime}, t_{\alpha}\right) .
$$

where the summation is taken over all $i, \alpha$ with $1 \leqq i<n$ and $|\alpha|=i, \alpha \in J$.

4. Compactness of the imbedding operator. In this section, we shall obtain some results analogous to the following well known fact (see [5], p. 75) in the usual Sobolev spaces regarding weak convergence:

(P) If $\left\{z_{k}\right\}$ is a sequence of functions in $W_{p}^{n}(G), p>1$ converging weakly to some $z \in W_{p}^{n}(G)$ then $z_{k} \rightarrow z$ uniformly in $G$ and $D_{\alpha} z_{k} \rightarrow D_{\alpha} z$ strongly in $L_{p}(G)$ for all multiindices $\alpha$ with $0 \leqq|\alpha|<n$ (need not be in $\bar{J})$. The same conclusion holds for $p=1$ provided in addition the set $\left\{D_{\alpha} z_{k}|| \alpha \mid=n, k=1,2, \cdots\right\}$ is equiabsolutely integrable.

It is to be noted that the above statement is not valid in $W_{p}^{*}(G)$ as the following examples show:

EXAMPLE 1. Let

$$
G=[(x, y) \mid 0 \leqq x \leqq 1,0 \leqq y \leqq 1] \text {. }
$$

For $k=1,2, \cdots$ and $i=0, \cdots, k-1$, let

$$
I_{i k}=[(x, y) \in G \mid(i / k) \leqq x \leqq(2 i+1) / 2 k]
$$

and

$$
I_{i k}^{\prime}=[(x, y) \in G \mid(2 i+1) / 2 k \leqq x \leqq(i+1) / k]
$$

and let $z_{k}(x, y)$ be defined on $G$ by $z_{k}(x, y)=y(x-i / k)$ in $I_{i k}$ and $=y(-x+(i+1) / k)$ in $I_{i k}^{\prime}, i=0, \cdots,(k-1)$. Then $z_{k}$ is continuous and belongs to $W_{p}^{*}(G), p \geqq 1$; the derivatives $z_{k x}, z_{k y}, z_{k x y}$ are given by $z_{k x}=y ; z_{k y}=x-i / k ; z_{k x y}=+1$ in $I_{i k} ; z_{k x}=-y ; z_{k y}=-x+(i+1) / k$; $z_{k x y}=-1$ in $I_{i k}^{\prime}, i=0, \cdots,(k-1)$. Clearly, $z_{k} \rightarrow 0$ weakly in $W_{p}^{*}(G)$, $p \geqq 1$ and $z_{k x y} \rightarrow 0$ weakly in $L_{p}(G)$, while $z_{k y}$ and $z_{k}$ converge uniformly to 0 . But $z_{k x}$ has norm $(p+1)^{-1 / p}$ for each $k$ and as such does not converge to 0 strongly in $L_{p}(G)$. It is to be noted that $z_{k} \notin W_{p}^{2}(G)$ since the generalized derivative $z_{k x x}$ does not exist.

\section{EXAMPLE 2. Let}

$$
G=[(x, y) \mid-\pi \leqq x \leqq \pi ;-\pi \leqq y \leqq \pi] .
$$

Let $z_{k}(x, y)=(\sin k x+\sin k y) / k$. Then $z_{k x}=\cos k x: z_{k y}=\cos k y$ and $z_{k x y}=0 ; k=1,2, \cdots$. Clearly, $z_{k} \in W_{2}^{*}(G)$ and $z_{k x y} \rightarrow 0$ weakly. But 
$z_{k x} \rightarrow 0, z_{k y} \rightarrow 0$ weakly and not strongly. However, $z_{k} \rightarrow 0$ uniformly in $G$. It is to be observed that $z_{k x}$ and $z_{k y}$ do not converge pointwise.

More generally, if $I$ is any interval on the real line, if $\left\{\varphi_{k}(x)\right\}$ is an orthogonormal sequence in $L_{2}(I)$ and if $G=I \times I$, then $z_{k}(x, y)=\varphi_{k}(y)+\varphi_{k}(y)$ will be in $W_{2}^{*}(G)$ and converge to 0 uniformly as well as weakly in $W_{2}^{*}(G)$; but the derivatives $z_{k x}$ and $z_{k y}$ do not converge strongly.

Now, the following theorem valid in $W_{p}^{*}(G), p>1$ is analogous to statement $(P)$ :

\section{THEOREM 2. Let}

$$
G=[a, a+h]=\left\{t \mid t=\left(t_{1}, \cdots, t_{n}\right), a_{i} \leqq t_{\imath} \leqq a_{i}+h_{i}, i=1, \cdots, n\right\} .
$$

If $\left\{z_{k}\right\}$ is a sequence in $W_{p}^{*}(G), p>1$ weakly convergent to an element $z$ in $W_{p}^{*}(G)$, and if $z_{k}\left(\alpha_{\imath}, t_{\imath}^{\prime}\right) \rightarrow z\left(\alpha_{i}, t_{\imath}^{\prime}\right)$ uniformly in $G_{i}^{\prime}$ for $i=1, \cdots, n$ then $z_{k}(t) \rightarrow z(t)$ uniformly in $G$.

REMARK. Here $G_{\imath}^{\prime}=\left\{t_{i}^{\prime} \mid t_{i}^{\prime}=\left(t_{1}, \cdots, t_{\imath-1}, t_{i+1}, \cdots, t_{n}\right) \in\left[a_{i}^{\prime}, a_{\imath}^{\prime}+h_{i}^{\prime}\right]\right\}$ as in no. 2.

Proof. Weak convergence of $z_{k}$ to $z$ in $W_{p}^{*}(G)$ implies in particular, weak convergence of the highest order derivatives $D^{n} z_{k}$ to $D^{n} z$ in $L_{p}(G), p>1$. This implies in turn the pointwise convergence of $w_{k}(t)=\int_{a}^{t} D^{n} z_{k}(s) d s$ to $w(t)=\int_{a}^{7} D^{n} z(s) d s$. In view of the formula (3.11) and the hypothesis, this yields that $z_{k}(t) \rightarrow z(t)$ a.e. in G. But since weak convergence of $D^{n} z_{k}$ to $D^{n} z$ in $L_{p}(G), p>1$ implies boundedness of the norms of $\left\{D^{n} z_{k}, k=1,2, \cdots\right\}$, if follows that the sequence $\left\{w_{k}(t)\right\}$ is equicontinuous and equibounded. It follows that the sequence $z_{k}$ is uniformly convergent to $z$. Indeed, if $z_{k}\left(t_{0}\right) \rightarrow z\left(t_{0}\right)$ for some fixed $t_{0} \in G$, then

$$
\left|z_{k}(t)-z(t)\right| \leqq\left|z_{k}(t)-z_{k}\left(t_{0}\right)\right|+\left|z_{k}\left(t_{0}\right)-z\left(t_{0}\right)\right|+\left|z\left(t_{0}\right)-z(t)\right| .
$$

Also, since $\left\{w_{k}(t)\right\}$ are equicontinuous and $z_{k}\left(a_{i}, t_{i}^{\prime}\right)$ is uniformly convergent, it follows that $\left\{z_{k}, z, k=1,2, \cdots\right\}$ is equicontinuous. This fact along with the above inequalities yields uniform convergence of $z_{k}$ to $z$ in a neighborhood of $t_{0}$. But since $G$ is bounded (and closed), the same conclusion is valid through $G$. Thus, $z_{k} \rightarrow z$ in $C(G)$.

REMARKs. 1. Above theorem is valid for $p=1$ provided $D^{n} z_{k}$, $k=1,2, \cdots$ is assumed to be equiabsolutely integrable. The proof remains the same.

2. In view of the remarks in no. 3 , the above hypothesis of 
uniform convergence of $z_{l k}\left(a_{i}, t_{i}^{\prime}\right)$ can be replaced by weak convergence of $D_{\alpha} z_{k}\left(a_{\alpha}, t_{\alpha}^{\prime}\right)$ to $D_{\alpha} z\left(a_{\alpha}, t_{\alpha}^{\prime}\right)$ in $L_{p}\left(G_{\alpha}^{\prime}\right)$ for $\alpha, 0 \leqq|\alpha|<n$ and $p>1$. For $p=1$, we assume in addition that

$$
\left\{D_{\alpha} z_{k}\left(a_{\alpha}, t_{\alpha}^{\prime}\right)|k=1,2, \cdots ; 0 \leqq| \alpha \mid<n, \alpha \in \bar{J}\right\}
$$

is equiabsolutely integrable.

3. The above hypothesis on the boundary values of $z_{k}$ and $D_{\alpha} z_{k}$ are all automatically satisfied in many applications. For example, in a Darboux problem, the boundary values are prescribed apriori so that $z_{k}\left(a_{\imath}, t_{\imath}^{\prime}\right)=\varphi_{\imath}\left(t_{\imath}^{\prime}\right)$ and the required convergence is obviously satisfied.

4. The hypothesis of uniform convergence of $z_{k}\left(\alpha_{\imath}, t_{2}^{\prime}\right)$ can be replaced by that of $z_{k}\left(\bar{t}_{i}, t_{\imath}^{\prime}\right)$ where $\bar{t}$ is a fixed element of $G$.

5. Integro-differential operators. Weak convergence in $W_{p}^{*}(G)$ as usual implies weak convergence in $L_{p}(G)$ of each of the derivatives $D_{\alpha} z_{k}, \alpha \in \bar{J}$, to $D_{\alpha} z$. However, weak convergence in $W_{p}^{*}(G)$ does not imply the following condition on weak convergence on "lines of boundary":

(B) The sequence $z_{k} \in W_{p}^{*}(G)$ is said to converge in (B) to $z$ if for each $\alpha \in J$ (that is $\alpha_{i} \neq 1$ for some $i$ ), $D_{\alpha}\left(z_{k}-z\right)\left(\alpha_{\pi}, s_{\alpha}, t_{\alpha+\pi}^{\prime}\right)$ con vergences weakly in $L_{p}\left(G_{\alpha}\right)$ to zero, for almost all $t_{\alpha+\pi}^{\prime}$ in $G_{\alpha+\pi}^{\prime}$ with $\pi \in J, \quad 1 \leqq|\pi| \leqq n-|\alpha|, \quad \pi \cdot \alpha=0 ;$ [In particular, $D_{\alpha}\left(z_{k}-z\right)\left(\alpha_{\pi}, t_{\pi}^{\prime}\right)$ (with $s=0$ ) should converge weakly to zero].

EXAMPLE. Let $G=[0,1] \times[0,1]=\{(x, y) \mid 0 \leqq x, y \leqq 1\}$. Let $z_{k}(x, y)$ be defined on $G$ as Example 1, no. 4. Then, the deriatives of $z_{k}$ are given by $z_{k x}=y ; \quad z_{k y}=x-(i / k) ; \quad z_{k x y}=+1$ for $(i / k) \leqq x \leqq(2 i+1) / 2 k ; z_{k x}=-y ; z_{k y}=-x+(i+1) / k ; z_{k x y}=-1$ for $(2 i+1) / 2 k \leqq x \leqq(i+1) / k$; for $i=0, \cdots,(k-1)$.

In this case $z_{k x}(1, y)=-y$ and this certainly does not converge weakly to zero in $[0,1]$. Thus, $z_{k}$ does not coverge to zero in (B). However, $z_{k} \rightarrow 0$ weakly in $W_{p}^{*}(G)$.

REMARK. Condition (B) is certainly satisfied if there is a fixed function $\varphi(t), t \in \partial G$ with $z_{k}(t)=\varphi(t)$ for all $k$ and $t \in \partial G$.

The condition (B) is used in the following theorem which in turn is used in establishing closure properties of certain operators. We recall from no. 2 that for each $\alpha \in \bar{J}-\{0\}\left(\alpha_{\imath}=0\right.$ or 1 for all $i$, $\alpha_{i} \neq 0$ for some $\left.i\right) t_{\alpha}$ denotes the $|\alpha|$-vector obtained by omitting those coordinates $t_{\nu}$ in $\left(t_{1}, \cdots, t_{\nu}\right)$ for which $\alpha_{i}=0$. Let $G_{\alpha}=\left\{t_{\alpha} \mid a_{\alpha} \leqq t_{\alpha} \leqq a_{\alpha}+h_{\alpha}\right\}$.

THEOREM 3. Let $z_{k}, z \in W_{p}^{*}(G), 1 \leqq p \leqq \infty, k=1,2, \cdots$, and let 
$z_{k} \rightarrow z$ weakly in $W_{p}^{*}(G)$ as $k \rightarrow \infty$. Let condition (B) hold. Let $R_{\alpha}(t)$ be a given element of $L_{q}(G)$ with $\alpha \in J$ and $1 / p+1 / q=1$ (with the usual convention that $q=\infty$ if $p=1$ and vice versa). Let there exist functions $K_{\alpha}\left(t_{\alpha}\right) \in L_{q}\left(G_{\alpha}\right)$ such that $\left|R_{\alpha}\left(t_{\alpha}^{\prime}, t_{\alpha}\right)\right| \leqq K_{\alpha}\left(t_{\alpha}\right)$ for almost all $t_{\alpha}^{\prime} \in G_{\alpha}^{\prime}$. Then the sequences $T_{\alpha} R_{\alpha} D_{\alpha} z_{k}=\int_{a_{\alpha}}^{t_{\alpha}} R_{\alpha}\left(t_{\alpha}^{\prime}, s_{\alpha}\right) D_{\alpha} z_{k}\left(t_{\alpha}^{\prime}, s_{\alpha}\right) d s_{\alpha}$ belong to $L_{p}(G)$ and converge in measure to $T_{\alpha} R_{\alpha} D_{\alpha} z, \alpha \in J$. If $p>1$, then this convergence is also in $L_{r}$ norm, $1 \leqq r<p$.

Proof. (i ) For $z \in W_{p}^{*}(G)$ and $\alpha \in J, D_{\alpha} z \in L_{p}(G)$ and

$$
\begin{aligned}
\int_{G} \mid & \left.T_{\alpha} R_{\alpha} D_{\alpha} z\right|^{p} d t=\int_{G}\left(\int_{a_{\alpha}}^{t_{\alpha}} R_{\alpha} D_{\alpha} z\right)^{p} d t \\
& \leqq \int_{G}\left(\int_{a_{\alpha}}^{t_{\alpha}}\left|R_{\alpha}\left(s_{\alpha}, t_{\alpha}^{\prime}\right)\right|^{q}\right)^{p / q} \cdot\left(\int_{a_{\alpha}}^{t_{\alpha}}\left|D_{\alpha} z\left(a_{\alpha}^{\prime}, s_{\alpha}\right)\right|^{p}\right) d t \\
& \leqq \int_{G}\left(\int_{a_{\alpha}}^{t_{\alpha}}\left|K_{\alpha}\left(s_{\alpha}\right)\right|^{q}\right)^{p / q}\left(\int_{a_{\alpha}}^{t_{\alpha}}\left|D_{\alpha} z\left(s_{\alpha}, t_{\alpha}^{\prime}\right)\right|^{p}\right) d t \\
& \leqq \int_{a_{\alpha}}^{a_{\alpha}+h_{\alpha}} \int_{a_{\alpha}^{\prime}}^{a_{\alpha}^{\prime}+h_{\alpha}^{\prime}}\left(\int_{a_{\alpha}}^{a_{\alpha}+h_{\alpha}}\left|K_{\alpha}\left(s_{\alpha}\right)\right|^{q}\right)^{p / q} \cdot\left(\int_{a_{\alpha}}^{t_{\alpha}}\left|D_{\alpha} z\left(s_{\alpha}^{\prime}, t_{\alpha}\right)\right|^{p}\right) \\
& \leqq h_{\alpha}\left(\left\|K_{\alpha}\right\|_{q} \cdot\left\|D_{\alpha} z\right\|_{p}\right)^{p} .
\end{aligned}
$$

If $p=1, q=\infty, R_{\alpha} \in L_{\infty}$ say $\left|R_{\alpha}\right| \leqq M_{\alpha}$ then

$$
\int_{G}\left|T_{\alpha} R_{\alpha} D_{\alpha} z\right| \leqq M_{\alpha} \int_{G}\left|T_{\alpha} D_{\alpha} z\right| \leqq M_{\alpha} h_{\alpha}\left\|D_{\alpha} z\right\|
$$

This proves that $T_{\alpha} R_{\alpha} D_{\alpha} z \in L_{p}(G)$.

(ii) If $z_{k} \rightarrow z$ weakly in $W_{p}^{*}(G)$ then $T_{\alpha} R_{\alpha} D_{\alpha} z_{k} \rightarrow T_{\alpha} R_{\alpha} D_{2} z$ a.e. in $G$. Indeed, using (3.10) with $z$ replaced by $z_{k}-z$, we get

$$
\begin{aligned}
& \left|\int_{a_{\alpha}}^{t_{\alpha}} R_{\alpha}\left(s_{\kappa}, t_{\alpha}^{\prime}\right) D_{\alpha}\left(z_{l_{k}}-z\right)\left(s_{\alpha}, t_{\alpha}^{\prime}\right)\right| \\
& \quad \leqq\left|\int_{a_{\alpha}}^{t_{\alpha}} R_{\alpha}\left(s, t_{\alpha}^{\prime}\right)\left(\int_{a_{\alpha}^{\prime}}^{t_{\alpha}^{\prime}} D^{n}\left(z_{k}-z\right)\left(s_{\alpha}, s_{\alpha}^{\prime}\right) d s_{\alpha}^{\prime}\right) d s_{\alpha}\right| \\
& \left.\quad+\mid \int_{a_{\alpha}}^{t_{\alpha}} R_{\alpha}\left(s_{\alpha}, t_{\alpha}^{\prime}\right) \Sigma(-1)^{i-1} D_{\alpha}\left(z_{k}-z\right)\left(a_{\pi}, s_{\alpha}, t_{\alpha+\pi}^{\prime}\right) d s_{\alpha}\right) \mid
\end{aligned}
$$

where the summation in the last term is taken over $i, \pi$ with $1 \leqq i \leqq n-|\alpha|$; and $\pi \in J,|\pi|=i, \pi \cdot \alpha=0$.

The first term tends to zero a.e. in $G$ since $D^{n}\left(z_{k}-z\right) \rightarrow 0$ weakly in $L_{p}(G)$. The second term tends to zero due to condition (B).

(iii) Since $G$ is of finite measure and $T_{\alpha} R_{\alpha} D_{\alpha} z_{k} \rightarrow T_{\alpha} R_{\alpha} D_{\alpha} z$ a.e. in $G$, it follows that convergence is also in measure.

(iv) Let us observe that (a) for $p>r \geqq 1$

$$
\begin{gathered}
\int_{L}\left|T_{\alpha} R_{\alpha} D_{\alpha} z_{k}\right|^{r} \leqq\left\|T_{\alpha} R_{\alpha} D_{\alpha} z_{k}\right\|^{r}(|\operatorname{meas}(E)|)^{(p-r) / p} \\
\leqq\left\|K_{\alpha}\right\|\left\|^{r} h_{\alpha}^{r / p}\right\| D_{\alpha} z_{k} \|^{r}(|\operatorname{meas}(E)|)^{(p-r) / p}
\end{gathered}
$$


and (b) $\left\{\left\|D_{\alpha} z_{k}\right\|, k=1,2, \cdots\right\}$ is bounded due to weak convergence of $D_{\alpha} z_{k}$. Thus, $\left\{T_{\alpha} R_{\alpha} D_{\alpha} z_{k}\right\}$ is a sequence in $L_{p}$ converging in measure to $T_{\alpha} R_{\alpha} D_{\alpha} z$ and such that $\int_{E}\left|T_{\alpha} R_{\alpha} D_{\alpha} z_{k}\right|^{r}, 1 \leqq r<p, k=1,2, \cdots$ is uniformly absolutely continuous. By using a theorem in $L_{r}$-convergence (see [6], p. 231, Theorem 32.2, and p. 235, Problem 32.f) it follows that $T_{\alpha} R_{\alpha} D_{\alpha} z_{k}$ converges to $T_{\alpha} R_{\alpha} D_{\alpha} z$ strongly in $L_{r}$ norm.

REMARK. Under the conditions of the Theorem 3 above, the operator $A z=\left(z, T_{\alpha} R_{\alpha} D_{\alpha} z\right)$ from $W_{p}^{*}(G)$ in to $\left(L_{p}(G)\right)^{2 n}, p>1$ takes weakly convergent sequences in to sequences with strongly convergent subsequences. In this case, $A$ is compact.

6. Closure properties in $W_{p}^{*}(G)$. We have seen that $W_{p}^{*}(G) \subset$ $C(G)$ for $1 \leqq p \leqq \infty$, and thus it is a subspace. However, $W_{p}^{*}(G)$ is not closed in $C(G)$. Indeed, for $n=1, G=[0,1]$, let $z_{k}(t)$ be a sequence of polynomials converging uniformly to the Cantor function $z(t)$ on $G$. Since $z(t)$ is not absolutely continuous, $z \notin W_{p}^{*}(G)$ while $z_{k} \in W_{p}^{*}(G)$, $k=1,2, \cdots$. This situation prompts us to consider closable and closed operators on $W_{p}^{*}(G)$. First, we shall follow Cesari and Kaiser [2], and make the following definitions:

Definition 1. Let $(X, \tau)$ be a topological space and $S \subseteq X$. Let $A: S \rightarrow B, B$ a Banach space. We say that $A$ is a weakly (strongly) closed operator if $x_{n} \in S, x_{n} \rightarrow x$ (in $\tau$ ), $x \in X$, and $A x_{n} \rightarrow y \in B$ weakly (strongly) implies $x \in S$ and $A x=y$.

Definition 2. Let $(X, \tau)$ be a topological space and $B$ a Banach space. Let $S \subset X$ and $A: S \rightarrow B$. We say that $A$ has weak (strong) convergence property if $x_{n}, x \in S, x_{n} \rightarrow x$ (in $\tau$ ) implies $A x_{n} \rightarrow A x$ weakly (strongly).

EXAMPLES. 1. Obvious from the definition of the norm in $W_{p}^{*}(G)$, $G \subset E^{n}, n \geqq 1, p \geqq 1$ that for $\alpha \in J$ (that is $\alpha_{i}=0$ or 1 for all $i$ ) the operator $D_{\alpha}$ from $W_{p}^{*}(G)$ into $L_{p}(G)$ has the weak (and strong) convergence properties provided the topology $\tau$ of $W_{p}^{*}(G)$ is the norm topology.

2. $X=C(G)$, the space of contiuous functions on

$$
G=\left[t \in E^{n} \mid a_{i} \leqq t_{i} \leqq a_{i}+h_{i}\right]
$$

with uniform topology $\tau$. Let $S=W_{p}^{*}(G)$ and

$$
B=L_{p}(G) \times \prod_{0 \leqq|\alpha|<n} L_{p}\left(G_{\alpha}^{\prime}\right)
$$

and 


$$
A z=\left(D^{n} z, D_{\alpha} z\left(a_{\alpha}, t_{\alpha}^{\prime}\right)\right) .
$$

Then $A$ is a weakly (and hence strongly) closed operator from $S$ into $B$ for $p>1$. In fact, $A z_{k} \rightarrow A z$ weakly implies $z_{k} \rightarrow w$ uniformly (see Theorem 2 and Remark 2 of no. 4) where

$$
w=\int_{a}^{t} D^{n} z+\sum(-1)^{i-1} D_{\alpha} z\left(a_{\alpha}, t_{\alpha}^{\prime}\right)
$$

and the summation is over all $i, \alpha$ with $1 \leqq i<n,|\alpha|=i, \alpha \in J$. Also, $w \in W_{p}^{*}(G)$. Now $z_{k} \rightarrow z$ in $C(G)$ and $z_{k} \rightarrow w$ also in $C(G)$ implies $z=w$ and hence proof.

3. Let $X=C(G), \quad G=\left[t \in E^{n} \mid a_{i} \leqq t_{i}+h_{i}\right]=[a, a+h], \quad S=$ $\left\{z \in W_{p}^{*}(G) \mid z(t)=0\right.$ for $\left.t \in \partial G\right\} ; \quad B=L_{p}(G), p>1$. Then $A z=D^{n} z=$ $\partial^{n} z / \partial t_{1} \cdots \partial t_{n}$ is a weakly (and hence strongly) closed operator from $S$ in to $B$.

4. If $X=W_{p}^{1}(G), \quad p>1, \quad G=[a, a+h] \subset E^{n}$ and $S=$ $\left\{z \in W_{p}^{*}(G) \mid z(t)=0, t \in \partial G\right\}$ and $\tau$ is the weak topology; $B=L_{p}(G)$ and $A z=D^{n} z$; then $A$ is not necessarily closed; The lower order derivatives may not converge in the $L_{p}$ norm.

5. Let $X=W_{p}^{*}(G), G=[a, a+h] \subset E^{n}, p>1, \tau$ is the weak topology, $S_{\varphi}=\{z \in X \mid z(t)=\varphi(t)\}$ for $t \in \partial G$ and let $B=L_{r}(G)$. Let $A z=f\left(t, z, \int_{a_{\alpha}}^{t_{\alpha}} R_{\alpha} D_{\alpha} z\right)$ where $R_{\alpha}\left(t_{\alpha}\right)$ are functions of $t_{\alpha}$ alone and are elements of $L_{q}\left(G_{\alpha}\right)$ and where $f=f(t, \theta)$ is a real valued function defined on $G \times E^{2 n}, f$ is continuous in $t$, Lipschitzian in $\theta$, and $f(t, 0) \in L_{r}(G)$. Then $A$ has strong (and hence weak) covergence properties for $1 \leqq r<p$ while $A$ is weakly closed for $r=p$. Indeed, if $z_{k} \rightarrow z$ weakly in $W_{p}^{*}(G), p>1$ then we have by Theorem 3, no. 5, that $A z_{k} \rightarrow A z$ strongly in $L_{r}, 1 \leqq r<p$. This proves the convergence properties. If $r=p$ then $A z_{k}$ need not converge to $A z$. But if $A z_{k} \rightarrow w$ weakly for some $w \in\left(L_{p}(G)\right)^{2^{n}}$ then $A z_{k} \rightarrow w$ weakly also in $\left(L_{s}(G)\right)^{2^{n}}$ for $1 \leqq s<p$. But $A z_{k} \rightarrow A z$ strongly (and hence weakly) in $\left(L_{s}(G)\right)^{2^{n}}$. Thus, $A z=w$ and weak closure of $A$ is proved.

An example of a function $f$ satisfying the requirements above is $f(t, x, y)=e^{t} \sin x \cos y, t \in G=[0,1]$ and $(x, y) \in E^{2}$.

7. A Darboux problem. In this section, we shall obtain an existence theorem for solutions of a Darboux problem with integrodifferential system of equations. We shall follow notations in no. 5 . Thus, $G=\left[t \in E^{n} \mid t=\left(t_{1}, \cdots, t_{n}\right), a_{i} \leqq t_{i} \leqq a_{i}+h_{i}, i=1, \cdots, n\right]$ and $J$ denotes $\left\{\alpha=\left(\alpha_{1}, \cdots, \alpha_{n}\right) \mid \alpha_{i}=0\right.$ or 1 for all $i, \alpha_{i} \neq 1$ for some $\left.i\right\}$. Also $\bar{J}=J \cup\{(1, \cdots, 1)\}$. For $\alpha \in \bar{J}-\{0\}, G_{\alpha}=\left\{t_{\alpha} \mid a_{\alpha} \leqq t_{\alpha} \leqq a_{\alpha}+h_{\alpha}\right\}$ where $t_{\alpha}$ is obtained from $\left(t_{1}, \cdots, t_{n}\right)$ by deleting those $t_{i}$ for which $\alpha_{i}=0$. Let $\varphi_{\alpha}\left(t_{\alpha}\right)$ be a given element of $\left(W_{p}^{*}\left(G_{\alpha}\right)\right)^{\nu}, 1 \leqq p \leqq \infty$. Let $\varphi_{\alpha_{1}}\left(a_{\alpha_{2}}\right)=\varphi_{\alpha_{2}}\left(a_{\alpha_{1}}\right)$ for $\alpha_{1}, \alpha_{2} \in J-\{0\}$. Let $U$ be a fixed closed subset of 
$E^{m}$. Let $g=g(t, \zeta, u)=\left(g_{1}, \cdots, g_{\nu}\right)$ be defined on $G \times E^{\theta} \times U$ where $\theta=\left(2^{n}-1\right) \cdot 3 \nu$. Let $g$ take values in $E^{\nu}, \nu \geqq 1$. Let $g$ be measurable in $t$, continuous in $u$ and Lipchitzian in $\zeta$. Thus, there exists a constant $K>0$ such that

$$
\left|g(t, \zeta, u)-g\left(t, \zeta^{\prime}, u\right)\right| \leqq K\left|\zeta-\zeta^{\prime}\right|, \zeta, \zeta^{\prime} \in E^{\theta} .
$$

For $\alpha \in \bar{J}-\{0\}$ let $R_{\alpha}(t), R_{\alpha}^{\prime}(t)$ be $\nu \times \nu$ matrices with entries in $L_{q}(G), 1 / p+1 / q=1$. Further, let there exist functions $K_{\alpha}\left(t_{\alpha}\right)$ in $L_{q}\left(G_{\alpha}\right)$ and $K_{\alpha}^{\prime}\left(t_{\alpha}^{\prime}\right)$ in $L_{q}\left(G_{\alpha}^{\prime}\right)$ such that for $a \in J-\{0\},\left|R_{\alpha}(t)\right| \leqq K_{\alpha}\left(t_{\alpha}\right)$ for almost all $t_{\alpha}^{\prime} \in G_{\alpha}^{\prime}$ and $\left|R_{\alpha}^{\prime}(t)\right| \leqq K_{\alpha}^{\prime}\left(t_{\alpha}^{\prime}\right)$ for almost all $t_{\alpha} \in G_{\alpha}$; and for $\alpha=(1, \cdots, 1),\left|R_{\alpha}(t)\right| \leqq K_{\alpha}$, a constant and $R_{\alpha}^{\prime}$ is an arbitrary $L_{q}(G)$ function. With this notation, we may state the Darboux problem as follows: Given $u(t)=\left(u^{1}, \cdots, u^{m}\right)$ measurable on $G$, with values in $U$, find $z(t) \in\left(W_{p}^{*}(G)\right)^{\nu}$ satisfying

$$
\begin{aligned}
& D^{n} z^{i}(t)=g_{i}\left(t, z, A_{1} z, A_{2} z, u(t)\right) \\
& \left(A_{1} z_{\alpha}^{i}=D_{\alpha} z^{2}, \quad \alpha \in J-\{0\}\right. \\
& \left(A_{2} z\right)_{\alpha}^{2}=\left(T_{\alpha} R_{\alpha} D_{\alpha} z^{i}, T_{\alpha} R_{\alpha}^{\prime} D_{\alpha}^{\prime} z^{2}\right), \quad \alpha \in \bar{J}-\{0\} \\
& i=1, \cdots, \nu, z(t)=\left(z^{1}, \cdots, z^{\nu}\right), \quad u(t)=\left(u^{1}, \cdots, u^{m}\right), \quad t \in G
\end{aligned}
$$

with boundary data

$$
z\left(\alpha_{\alpha}^{\prime}, t_{\alpha}\right)=\varphi_{\alpha}\left(t_{\alpha}\right), \quad t_{\alpha} \in G_{\alpha} .
$$

In the equations (7.1) above for each $i,\left(A_{1} z\right)^{i}$ and $\left(A_{2} z\right)^{i}$ are vectors with components $\left(A_{1} z\right)_{\alpha}^{2}, \alpha \in J-\{0\}$ and $\left(A_{2} z\right)_{\alpha}^{2}, \alpha \in \bar{J}-\{0\}$ respectively. Also, $A_{i} z=\left(\left(A_{2} z\right)^{1}, \cdots,\left(A_{2} z\right)^{2}\right), i=1,2$.

By using a new set of variables and using the equations of no. 3, the Darboux problem stated above can be written in an equivalent form with integral equations as follows:

$$
\begin{aligned}
& \sigma_{1}(t)=T^{n} g(s, \sigma(s), u(s))+\Sigma(-1)^{2-1} \varphi_{\pi^{\prime}}\left(t_{\pi}^{\prime}\right) \\
& \sigma_{2, \alpha}(t)=T_{\alpha}^{\prime} g\left(t_{\alpha}, s_{\beta}, \sigma\left(s_{\beta}\right) u\left(s_{\beta}\right)\right)+\Sigma(-1)^{i-1} D_{\alpha} \varphi_{\pi^{\prime}}\left(t_{\pi}^{\prime}\right), \quad \alpha \in J-\{0\}, \\
& \sigma_{3, \alpha}(t)=\left(T_{\alpha} R_{\alpha} \sigma_{2, \alpha}(s), T_{\alpha} R_{\alpha}^{\prime} \sigma_{2, \alpha^{\prime}}(s)\right), \quad \alpha \in J-\{0\}, \\
& \sigma_{3, \alpha}(t)=\left(T_{\alpha} R_{\alpha} g, T_{\alpha} R_{\alpha}^{\prime} \sigma_{1}\right), \quad \alpha=(1, \cdots, 1)
\end{aligned}
$$

where the summations are taken over all $i, \pi$ with $1 \leqq i \leqq\left|\alpha^{\prime}\right|$ and $\pi \in \bar{J},|\pi|=i, \pi \cdot \alpha=0$. Also, $\sigma=\left(\sigma_{1}, \sigma_{2}, \sigma_{3}\right), \sigma_{2}$ is a vector with components $\sigma_{2, \alpha}^{2}, i=1, \cdots, \nu ; \alpha \in J-\{0\}$, and $\sigma_{3}$ is a vector with components $\sigma_{3, \alpha}^{2}, i=1, \cdots, \nu$ and $\alpha \in \bar{J}-\{0\}$ while $\sigma_{1}=\left(\sigma_{1}^{1}, \cdots, \sigma_{1}^{\nu}\right)$. The solutions of $(7.1,2)$ and those of $(7.3)$ correspond as follows: $\sigma_{1}=z: \quad \sigma_{2, \alpha}=D_{\alpha} z, \quad \alpha \in J-\{0\} \quad$ and $\sigma_{3, \alpha}=\left(T_{\alpha} R_{\alpha} D_{\alpha} z, T_{\alpha} R_{\alpha}^{\prime} D_{\alpha}^{\prime} z\right)$ for $\alpha \in \bar{J}-\{0\}$.

The integral equations (7.3) being of the form studied in [9] by the author, we obtain the existence and uniqueness of the solutions 
of the integral system and hence of the Darboux problem. More precisely, we state

THEOREM 4. With the above notation, let $u(t)$ be a measurable function on $G$ such that $s_{u}(t)=g(t, 0, u(t)) \in\left(L_{p}(G)\right)^{\nu}, 1 \leqq p \leqq \infty$. Then, there exists corresponding to $u$, a unique $z \in\left(W_{p}^{*}(G)\right)$, satisfying the boundary conditions $z\left(a_{\alpha}^{\prime}, t_{\alpha}\right)=\varphi_{\alpha}\left(t_{\alpha}\right)$ and whose generalized partial derivatives $D_{\alpha} z, \alpha \in \bar{J}$, satisfy the equation (7.1) a.e. in $G$. Furthermore, there exist constants $B_{1}, B_{2}$ depending only on $\left(h_{1}, \cdots, h_{n}\right), \quad p, k$ (the Lipschitz constant of $g$ ), $\left\|K_{\alpha}\right\|,\left\|K_{\alpha}^{\prime}\right\|$, $\alpha \in J-\{0\}$; and $\left\|R_{\alpha}^{\prime}\right\|, K_{\alpha}, \alpha=(1, \cdots, 1)$, such that

$$
\begin{gathered}
\sum_{\alpha \in J}\left\|D_{\alpha} z\right\|_{p} \leqq B_{1}\left[\sum_{\alpha \in J}\left(h_{\alpha}\right)^{1 / p}\left(\left\|D_{\alpha} \varphi_{\alpha}\right\|+2^{-1}\left\|\varphi_{\alpha}\right\|_{p}\right)+|h| \cdot\left\|S_{u}\right\|_{p}\right] \\
|z(t)| \leqq 2^{-1}\left[\sum_{\alpha \in J}\left\|\varphi_{\alpha}\right\|+B B_{2}|h|+e^{K|h|}\left(B+\int_{G}\left|S_{u}\right|\right)\right] \\
\left|D_{\alpha} z(t)\right| \leqq \theta_{\alpha}\left(t_{\alpha}\right)+B B_{2}, \quad \alpha \in J-\{0\}
\end{gathered}
$$

where

$$
B=\sum\left\|D_{\alpha} \mathscr{P}_{\alpha}\right\|_{p} h_{\alpha}^{1 / p}+K h_{1} \cdots h_{n}\left(\left\|\mathscr{P}_{\alpha}\right\|_{\infty}\right)+\int_{G}\left|S_{u}(s)\right| d s
$$

and

$$
\begin{array}{r}
\theta_{\alpha}\left(t_{\alpha}\right)=e^{K \mid h_{\alpha}^{\prime} \prime}\left(\left|D_{\alpha} \varphi_{\alpha}\left(t_{\alpha}\right)\right|+K\left|h_{\alpha}^{\prime} \| \varphi_{\alpha}\left(t_{\alpha}\right)\right|+\int_{a_{\alpha}^{\prime}}^{a_{\alpha}^{\prime}+h_{\alpha}^{\prime}}\left|S_{u}\left(t_{\alpha}, t_{\alpha}^{\prime}\right)\right| d t_{\alpha}^{\prime}\right. \\
\alpha \in J-\{0\}
\end{array}
$$

REMARK 1. Since $g$ is Lipchitzian, we observe that

$$
\begin{aligned}
\left\|D^{n} z\right\|_{p} & =\left\|g\left(t, z, A_{1} z, A_{2} z, u\right)\right\|_{p} \\
& \leqq\|g(t, 0, u)\|+K\left(\|z\|+\left\|A_{1} z\right\|+\left\|A_{2} z\right\|\right) .
\end{aligned}
$$

Thus, the inequality (7.4) can be written as

$$
\|z\|^{*}=\sum_{\alpha \in J}\left\|D_{\alpha} z\right\|_{p} \leqq B_{3}\left[\left\|S_{u}\right\|+\sum_{\alpha \in J}\left\|\varphi_{\alpha}\right\|\left\|D_{\alpha} \varphi_{\alpha}\right\|\right]
$$

where $\|\cdot\|^{*}$ denotes the norm in $W_{p}^{*}(G)$; and where $B_{3}$ is a constant depending only on $h, K, p$ and the functions $R_{\alpha}, R_{\alpha}^{\prime}$.

REMARK 2. Let $z^{\prime}, z^{\prime \prime}$ denote solutions of $(7.1,2)$ corresponding to data $\varphi_{\alpha}^{\prime}, \phi_{\alpha}^{\prime \prime}$ in $\left(W_{p}^{*}\left(G_{\alpha}\right)\right)^{\nu}, \alpha \in J$ and control functions $u^{\prime}, u^{\prime \prime}$ respectively. Let $z=z^{\prime}-z^{\prime \prime} ; \varphi_{\alpha}=\varphi_{\alpha}^{\prime}-\varphi_{\alpha}^{\prime \prime} ; S(t)=\mid g\left(t, z^{\prime}, A_{1} z^{\prime}, A_{2} z^{\prime}, u^{\prime}\right)-$ $g\left(t, z^{\prime \prime}, A_{1} z^{\prime \prime}, A_{2} z^{\prime \prime}, u^{\prime \prime}\right) \mid$. With this notation, the above inequalities (7.4-6) hold with $S_{u}$ replaced by $S$. In particular, if $\varphi_{\alpha}^{\prime}=\phi_{\alpha}^{\prime \prime}$, for all $\alpha$ the inequality shows the behaviour of $z$ with respect to $u$. 
Illustration. We shall illustrate the above theorem in the case $n=2$. Let $G=[a, a+h] \times[b, b+k]$. Let $\varphi(x)$ and $\psi(y)$ be absolutely continuous functions for $x \in[a, a+h]$ and $y \in[b, b+k]$. The function $g(x, y, \zeta, u)$ is defined on $G \times E^{6} \times U$. Let $R(x, y)$, $S(x, y), T(x, y)$ be given functions in $L_{q}(G), 1 / p+1 / q=1$. Let there exist functions $K_{1}(x) \in L_{q}([a, a+h]), K_{2}(y) \in L_{q}([b, b+k])$, such that $|S(x, y)| \leqq K_{1}(x)$ for almost all $y$ and $|T(x, y)| \leqq K_{2}(y)$ for almost all $x$. Then, the above theorem states that if $u(x, y)$ is a measurable function such that $s_{u}(x, y)=|g(x, y, 0, u(x, y))| \in L_{p}(G), 1 \leqq p \leqq \infty$, then there exists a uique $z \in W_{p}^{*}(G)$, (corresponding to $u$ ), satisfying the boundary conditions $z(a, y)=\psi(y) ; z(x, b)=\varphi(x)$ and whose generalized derivatives $z_{x}, z_{y}, z_{x y}$ exist and satisfy the equation

$$
z_{x y}(x, y)=g\left(x, y, z, z_{x}, z_{y}, \int_{a}^{x} \int_{b}^{y} R z, \int_{a}^{x} S z_{x}, \int_{b}^{y} T z_{y}, u(x, y)\right) \text { a.e. in } G \text {. }
$$

REMARK. This type of equations have been studied by R. H. J. Germay [3].

\section{EXAMPLES.}

1. Let $G=[0,1] \times[0,1]$ and $U=[-1,1]$. Let $\varphi(x)=\psi(y)=0$; $R(x, y)=S(x, y)=T(x, y)=0$; Let $g=\zeta_{2}+x \tan u$ so that differential equation is $z_{x y}=z_{x}+x \tan u$ with boundary conditions $z(x, 0)=$ $z(0, y)=0$. By integration, the solution of this equation is seen to be $z(x, y)=e^{-y} \int_{0}^{x} \int_{0}^{y} e^{\beta} \alpha \tan u(\alpha, \beta) d \alpha d \beta$. In this case, the constants $B_{2}$ and $B_{3}$ of the above proposition are both equal to $e$.

2. Let $G=[0,1] \times[0,1]$ as before. Let $C($.) denote the Cantor function on $[0,1]$ (or any other function whose derivative exists almost everywhere but which is not absolutely continuous). Let us consider the equation $z_{z y}=0$ a.e. in $G$ with side conditions $z(x, 0)=$ $\int_{0}^{x} C(\alpha) d \alpha$ for $x \in[0,1]$ and $z(0, y)=\int_{0}^{y} C(\beta) d \beta$ for $y \in[0,1]$. It is readily seen that the solution of this equation is

$$
z(z, y)=z(x, 0)+z(0, y)=\int_{0}^{x} C(\alpha) d \alpha+\int_{0}^{y} C(\beta) d \beta .
$$

It is to be noted that this equation cannot have any solutions in $W_{p}^{2}(G)$, even though $C($.) possesses ordinary derivatives (equal to zero). Indeed, since $C($.$) is not absolutely continuous, it does not$ have a generalized derivative and consequently $z_{x}$ and $z_{y}$ do not have the corresponding generalized derivatives $z_{x x}$ and $z_{y y}$. However, the above solution belongs to $W_{p}^{*}(G)$ and the constants $B_{2}$ and $B_{3}$ are $=1$ in this case.

3. Let $G=[0,1] \times[0,1]$ and $U=E^{2}$. Let $a_{i}, b_{i}, i=1, \cdots, 7$ 
be some positive constants and $\varphi_{i}(x), \psi_{2}(y), i=1,2$ be some absolutely continuous functions. Let us consider the system of equations

$$
\begin{aligned}
z_{x y}(x, y)=[ & a_{1} x^{-1 / 2}+a_{2} z+a_{3} \sin \left(x z_{y}\right)+a_{4} \cos \left(y z_{x}\right) \\
& +a_{5} \int_{0}^{x} \varphi_{1}(\alpha) w_{x}(\alpha, y) d \alpha+a_{6} \int_{0}^{y} \psi_{1}(\beta) w_{y}(x, \beta) d \beta \\
& \left.+a_{7} \int_{0}^{x} \int_{0}^{y} \varphi_{1}(\alpha) \psi_{1}(\beta) w(\alpha, \beta) d \alpha d \beta\right] \sin u(x, y) \\
w_{x y}(x, y)= & {\left[b_{1} y^{-1 / 2}+b_{2} w+b_{3} \cos \left(x w_{y}\right)+b_{4} \sin \left(y w_{x}\right)\right.} \\
& +b_{5} \int_{0}^{x} \varphi_{2}(\alpha) z_{x}(\alpha, y) d \alpha+b_{6} \int_{0}^{y} \psi_{2}(\beta) z_{y}(x, \beta) d \beta \\
& \left.+b_{7} \int_{0}^{x} \int_{0}^{y} \varphi_{2}(\alpha) \psi_{2}(\beta) z(\alpha, \beta) d \alpha d \beta\right] \cos v(x, y)
\end{aligned}
$$

with side conditions $z(x, 0)=z(0, y)=w(x, 0)=w(0, y)=0 . \quad$ The solution exists in $W_{p}^{*}(G), 1 \leqq p<2$, for any measurable function $(u, v)$; because $g(x, y, 0, u, v)=\left(a_{1} x^{-1 / 2} \sin u, a_{2} y^{-1 / 2} \cos v\right)$ is in $L_{p}(G)$ for $1 \leqq p<2$. Also there are constants $C_{1}, C_{2}, C_{3}$ depending only on $a_{\imath}, b_{\imath}, i=1, \cdots, 7$ and $\varphi_{i}, \psi_{i}, i=1,2$ such that $|w(x, y)|,|z(x, y)|$, $\|\boldsymbol{z}\|^{*}, \quad\|w\|^{*} \leqq C_{1}, \quad(x, y) \in G, \quad\left|w_{x}(x, y)\right|, \quad\left|z_{x}(x, y)\right| \leqq a_{1} x^{-1 / 2}+C_{2}$; $\left|w_{y}(x, y)\right|,\left|z_{y}(x, y)\right| \leqq b_{1} y^{-1 / 2}+C_{3}$.

\section{REFERENCES}

1. L. Cesari, Problems of Optimization, vol. I, II, Springer-Verlag, to appear.

2. L. Cesari and P. J. Kaiser, Closed operators and existence theorems in multi-dimensional problems of calculus of variations, Bull. Amer. Math. Soc., 80 (1974), 473-478.

3. R. H. J. Germay, Sur les equations integrales, Memoires Soc. Roy. So. Liege, ser. 3 , 13, fasc 4, (1926), 1-26.

4. L. Hormander, Linear Partial Differential Operators, Grundlehren der Math. Wiss. Band 116, Springer-Verlag, Heidelberg, 1963.

5. C. B. Morrey, Multiple Integrals in the Calculus of Variations, Springer-Verlag, New York, N.Y., 1966.

6. M. E. Munroe, Introduction to Measure and Integration, Addison-Wesley, Reading, Mass., 1953.

7. G. Pulvirenti, Existence theorems for an optimal control problem relative to a linear hyperbolic partial differential equation, J. Optimation Theory and Appl., 7 (1971).

8. S. L. Sobolev, Applications of Functional Analysis in Mathematical Physics, vol. 7, Transactions of Matematical Monographs, American Mathematical Society, Providence, R. I., 1963.

9. M. B. Suryanarayana, On multidimensional equations of Volterra type, Pacific J. Math., 41 (1972), 809-828.

10. - Necessary conditions for optimization problems with hyperbolic partial differential equations, SIAM J. on Control, 11 (1973).

11. - Existence theorems for optimization problems concerning hyperbolic partial differential equations, J. Optimization Theory and Appl., 15 No. 4, (1975).

Received February 27, 1974.

EASTERN Michigan University

YPSILANTI, MI 48197 


\section{PACIFIC JOURNAL OF MATHEMATICS}

\section{EDITORS}

RICHARD ARENS (Managing Editor)

University of California

Los Angeles, CA 90024

R. A. BEAUMONT

University of Washington

Seattle, WA 98105

C. C. Moore

University of California

Berkeley, CA 94720

\section{J. DugundJI}

Department of Mathematics

University of Southern California

Los Angeles, CA 90007

R. Finn and J. Milgram

Stanford University

Stanford, CA 94305

\section{ASSOCIATE EDITORS}

\section{E. F. BECKENBACH}

B. H. NEUMANN

F. WoLF

K. YOSHIDA

\section{SUPPORTING INSTITUTIONS}

\author{
UNIVERSITY OF BRITISH COLUMBIA \\ CALIFORNIA INSTITUTE OF TECHNOLOGY \\ UNIVERSITY OF CALIFORNIA \\ MONTANA STATE UNIVERSITY \\ UNIVERSITY OF NEVADA \\ NEW MEXICO STATE UNIVERSITY \\ OREGON STATE UNIVERSITY \\ UNIVERSITY OF OREGON \\ OSAKA UNIVERSITY
}

\author{
UNIVERSITY OF SOUTHERN CALIFORNIA \\ STANFORD UNIVERSITY \\ UNIVERSITY OF HAWAII \\ UNIVERSITY OF TOKYO \\ UNIVERSITY OF UTAH \\ WASHINGTON STATE UNIVERSITY \\ UNIVERSITY OF WASHINGTON \\ AMERICAN MATHEMATICAL SOCIETY
}

The Supporting Institutions listed above contribute to the cost of publication of this Journal, but they are not owners or publishers and have no responsibility for its content or policies.

Mathematical papers intended for publication in the Pacific Jaurnal of Mathematics should be in typed form or offset-reproduced, (not dittoed), double spaced with large margins. Please do not use built up fractions in the text of your manuscript. You may however, use them in the displayed equations. Underline Greek letters in red, German in green, and script in blue. The first paragraph or two must be capable of being used separately as a synopsis of the entire paper. Items of the bibliography should not be cited there unless absolutely necessary, in which case they must be identified by author and Journal, rather than by item number. Manuscripts, in triplicate, may be sent to any one of the editors. Please classify according to the scheme of Math. Reviews, Index to Vol. 39. All other communications should be addressed to the managing editor, or Elaine Barth, University of California, Los Angeles, California, 90024.

The Pacific Journal of Mathematics expects the author's institution to pay page charges, and reserves the right to delay publication for nonpayment of charges in case of financial emergency

100 reprints are provided free for each article, only if page charges have been substantially paid. Additional copies may be obtained at cost in multiples of 50 .

The Pacific Journal of Mathematics is issued monthly as of January 1966. Regular subscription rate: $\$ 7200$ a year (6 Vols., 12 issues). Special rate: $\$ 36.00$ a year to individual members of supporting institutions.

Subscriptions, orders for back numbers, and changes of address should be sent to Pacific Journal of Mathematics, 103 Highland Boulevard, Berkeley, California, 94708.

PUBLISHED BY PACIFIC JOURNAL OF MATHEMATICS, A NON-PROFIT CORPORATION

Printed at Kokusai Bunken Insatsusha (International Academic Printing Co., Ltd.). 8-8, 3-chome, Takadanobaba, Shinjuku-ku, Tokyo 160, Japan.

Copyrit (C) 1975 by Pacific Journal of Mathematics Manufactured and first issued in Japan 


\section{Pacific Journal of Mathematics \\ Vol. 69, No. $2 \quad$ June, 1977}

Carol Alf and Thomas Alfonso O'Connor, Unimodality of the Lévy spectral

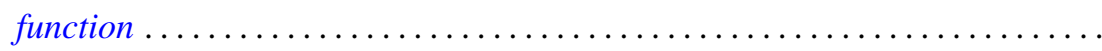

S. J. Bernau and Howard E. Lacey, Bicontractive projections and reordering of

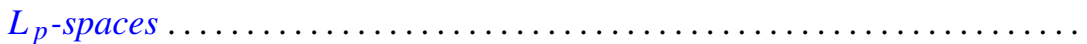

Andrew J. Berner, Products of compact spaces with bi-k and related spaces..... 303

Stephen Richard Bernfeld, The extendability and uniqueness of solutions of ordinary differential equations ...............................

Marilyn Breen, Decompositions for nonclosed planar m-convex sets ..........

Robert F. Brown, Cohomology of homomorphisms of Lie algebras and Lie

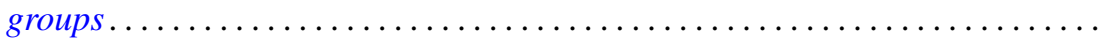

Jack Douglas Bryant and Thomas Francis McCabe, A note on Edelstein's iterative test and spaces of continuous functions ....................

Victor P. Camillo, Modules whose quotients have finite Goldie dimension ....... 333

David Downing and William A. Kirk, A generalization of Caristi's theorem with

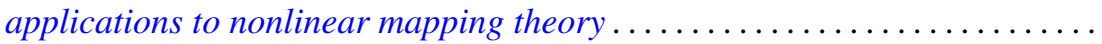

Daniel Reuven Farkas and Robert L. Snider, Noetherian fixed rings ...........

Alessandro Figà-Talamanca, Positive definite functions which vanish at

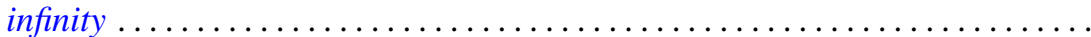

Josip Globevnik, The range of analytic extensions .................. 365

André Goldman, Mesures cylindriques, mesures vectorielles et questions de

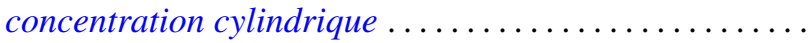

Richard Grassl, Multisectioned partitions of integers..........

Haruo Kitahara and Shinsuke Yorozu, A formula for the normal part of the

Laplace-Beltrami operator on the foliated manifold .... .

Marvin J. Kohn, Summability $R_{r}$ for double series .........

Charles Philip Lanski, Lie ideals and derivations in rings with involution ..

Solomon Leader, A topological characterization of Banach contractions . .

Daniel Francis Xavier O’Reilly, Cobordism classes of fiber bundles . .

James William Pendergrass, The Schur subgroup of the Brauer group . .

Howard Lewis Penn, Inner-outer factorization of functions whose Fourier series

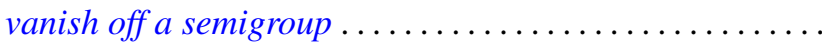

501

William T. Reid, Some results on the Floquet theory for disconjugate definite

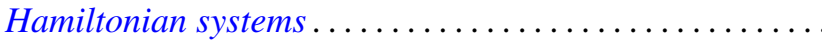

Caroll Vernon Riecke, Complementation in the lattice of convergence

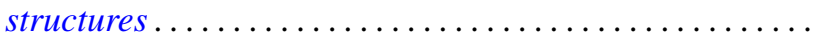

Louis Halle Rowen, Classes of rings torsion-free over their centers ......... 527

Manda Butchi Suryanarayana, A Sobolev space and a Darboux problem ....... 535

Charles Thomas Tucker, II, Riesz homomorphisms and positive linear maps.... 551

William W. Williams, Semigroups with identity on Peano continua ........... 557

Yukinobu Yajima, On spaces which have a closure-preserving cover by finite 